previous admissions and psychosocial assessments or referrals made were collected. Home postcodes were matched to local authority districts and mean index of multiple deprivation decile was calculated for that area, with 1 being the most deprived.

Results Two hundred and thirty-seven cases of violent injury were included; $94.5 \%$ of cases were male and the mean age was 19.4 years. The local authority district in which the highest number of victims resided had a mean index of multiple deprivation score of 3.7. Of all cases, only two patients received a full HEEADSSS assessment. 63.3\% of individuals received a form of psychosocial referral, and $15.9 \%$ of these referrals were to third sector organisations. $8 \%$ of victims had been previously stabbed. Of those that had presented to the same major trauma centre in the past due to a stabbing, none held a recorded HEEADSSS assessment and 25\% received no form of psychosocial referral nor intervention.

Conclusion There continues to exist a prevalence of youth violence predominantly against young males. HEEADSSS assessment methods can be optimised to improve yield and identify adolescents at higher risk of sustaining injury. Screening must be followed up with timely and appropriate innovative interventions, suited to individual social circumstances, to prevent reoccurrence of violent incidents.

Footnote Home, Education/Employment, Eating, Activities, Drugs, Sexuality, Suicidal ideation and Safety from injury and violence(HEEADSSS)

\section{G539(P) ARE WE REQUESTING TOO MANY STOOL CULTURES IN PAEDIATRICS? AN EXPERIENCE FROM PRIMARY AND SECONDARY CARE}

${ }^{1} S$ Fernandes Lucas, ${ }^{1} \mathrm{~A}$ Singh, ${ }^{1} \mathrm{D}$ Kunhanna, ${ }^{1} \mathrm{~F}$ Awadalla, ${ }^{2} \mathrm{~J}$ Ahmed. ${ }^{1}$ Paediatrics, Southend University Hospital NHS FT, Southend-on-Sea, UK; ${ }^{2}$ Microbiology, Southend University Hospital NHS FT, Southend-on-Sea, UK

\subsection{6/archdischild-2020-rcpch.457}

Aims Finding the causative organism does not usually change the management of acute infective gastroenteritis, hence investigations such as stool culture should only be performed in selective cases. We intended to find if General Practitioners and Paediatricians were requesting stool cultures appropriately as recommended by the NICE guidelines for the diagnosis and management of diarrhoea and vomiting caused by gastroenteritis in children under 5. The guidelines recommend the performance or consideration of performance of stool microbiological investigations in specific cases, for instance in the presence of blood and/or mucous in stool.

Methods Data was collected retrospectively from children under 5 years of age who had stool cultures performed over a period of 6 months (May 2018 to October 2018) in their local hospital or GP practice. The list of patients was provided by the laboratory and the information about the clinical indications and results was collected via the Information Technology system shared between primary and secondary care. The information about management was obtained from GP surgeries and hospital records.

Results A total of 334 stool samples (GP practices $=260$ and Hospital=74) were sent for culture. The requests were inappropriate for the majority of patients with an overall of $58 \%$
$(62 \%$ in community vs. $45 \%$ in hospital). Bacterial growths were observed in 7 samples (3 salmonella and 4 campylobacter). From all the appropriate requests (139), 4.3\% were positive for bacterial growth and $0.7 \%$ were treated with antibiotic therapy. From the inappropriately requested samples (195), 0.5\% were positive for bacterial growth with no patient receiving antibiotic treatment.

Conclusion A significant number of stool culture requests by primary and secondary care did not follow the NICE recommendations. Inappropriate requests were more prevalent in primary than secondary care. There is a scope to investigate the reason for this difference. The probability of diagnosing a bacterial growth in a stool culture was significantly increased by approximately 8 times where recommendations were followed. Requesting investigations appropriately would also decrease unnecessary workload and reduce the burden on NHS finances.

\section{G540(P) ADOLESCENT BREAST LUMPS: DEVELOPMENT OF A ONE STOP PATHWAY}

SThompson, L Roe. Paediatrics, University Hospital North Midlands, Stoke-on-Trent,UK

\subsection{6/archdischild-2020-rcpch.458}

Introduction All post pubertal females presenting with breast lumps were previously sent to the paediatric oncology clinic via the 2 week wait pathway. They would be seen in clinic, examined and when a lump was confirmed an out patient ultrasound was then requested (on a separate day, usually a few weeks later). The young person would then re-attend the oncology clinic to get the results and management plan before finally being discharged. The whole process could take up to 6 weeks. Gp surgeries would usually advise families incorrectly that a scan would take place on the same day. When this then didn't happen this would lead to additional anxiety on top of the fear that their young person could have cancer (even though that was never the case).

Aims To streamline the process so that it resembled the adult one stop process (minus the biopsy) thereby alleviating fears and worries quickly and minimizing hospital visits.

Method An average 35 post pubertal females were referred in annually with breast lumps to the paediatric oncology clinic. Two female pediatricians with an interest in oncology met with adult breast surgeon and breast radiologist to agree a one stop pathway. One radiology slot identified at the start of the adult clinic that could be utilized by adolescent if lump identified in new patient clinic slot.

Results One stop pathway set up with patient information leaflet (Adolescent focused highlighting benign nature of these type of lumps) sent to families prior to appointment setting out process and likely outcome. Well evaluated by adolescents and families. No loss of income to trust

Conclusions An efficient pathway that reduces time to diagnosis and management plan thereby alleviating anxiety and reducing time out from school/off work (parents). Historically GP's assumed that scans would happen on the same day and so incorrectly advised patients. By matching the adult process this is avoided. Could similar one stop pathways be used for other adolescent conditions and transition pathways as they are in adults? 